DIGITALCOMMONS

@WAYNESTATE-
Michigan Journal of Counseling:

Research, Theory and Practice

Volume 31 | Issue 2

Article 2

$10-1-2003$

\title{
Color Consciousness and African American Adults: Self Perception, Trait Ascription, and Interpersonal Experiences
}

Alfiee M. Breland-Noble

Duke University Medical Center, abreland@psych.duhs.duke.edu

Wanda Collins

Michigan State University

Jennifer King

Texas A \& $M$ University

Follow this and additional works at: https://digitalcommons.wayne.edu/mijoc

\section{Recommended Citation}

Breland-Noble, A. M., Collins, W., \& King, J. (2003). Color Consciousness and African American Adults: Self Perception, Trait Ascription, and Interpersonal Experiences, Dimensions of Counseling, 31(2), 1-12. doi:10.22237/mijoc/1064966460

This Article is brought to you for free and open access by the Open Access Journals at DigitalCommons@WayneState. It has been accepted for inclusion in Michigan Journal of Counseling: Research, Theory and Practice by an authorized editor of DigitalCommons@WayneState. 
reconstruction and the civil rights movement, skin color is an issue that has remained salient for African Americans since they were first transported to America en masse. Of the existing empirical data in color consciousness, most is limited to a few areas, namely, socioeconomic status, ethnic identity, and self-esteem. The following brief review of the literature provides an overview of the aforementioned areas and context for the import of the current study.

\section{Skin Tone and Socioeconomic Status}

In the 1999 book, "Our Kind of People," Lawrence Otis Graham, an African American man, describes his childhood as one where he clearly understood, at a very early age, the importance of skin tone and socioeconomic status. He writes, "At age six, I already understood the importance of achieving a better shade of black." (p.1). He then describes the great historical and current distinctions regarding African American skin tone-based class stratification. Prior to the publication of this important book, Breland (1998) provided sociohistorical evidence of the origins of the class distinctions existent among African Americans. During slavery, European American enslavers afforded privileges to lighter skinned African descendants (who were often a product of their forced, coerced and/or consensual 'unions' with African women) that were not offered to the darker skinned "full" Africans (Keith \& Herring, 1991; OkazawaRey, Robinson, \& Ward, 1987; Sandler, 1994; Scales-Trent, 1995). Over time, these descendants came to have considerably lighter complexions than their fully African counterparts and with their European lineage, came to also have more privileges. The privileges were maintained through the abolition of the institution of slavery and provided the lighter skinned African Americans with the property, education, money and means to then establish themselves as members of a distinctly different socioeconomic group (Breland, 1998; Edwards, 1972; Graham, 1999; Hughes \& Hertel, 1990; Keith \& Herring, 1991; Maddox \& Gray, 2002; Seltzer \& Smith, 1991).

\section{Ethnic identity, Self Esteem and Skin Tone}

Researchers have suggested that African American ethnic identity and self esteem are connected to skin tone. Specifically, Hughes and Hertel (1990) and Keith and
Herring (1991) reported that darker skinned African Americans described more intense and frequent experiences of racism and discrimination than their lighter skinned counterparts. Further, their research suggested that lighter skinned African Americans hold weaker ties to their ethnic heritage than darker skinned African Americans. As disturbing as these trends may seem however, one should note that they were based on a few studies and as such should be interpreted with caution.

Coard, Breland and Raskin (2001) reported that a sample of darker skinned African American men who reported satisfaction with their skin tone also reported lower levels of self-esteem. Bond and Cash (1992) reported that for a sample of African American women, a majority believed that, "African American men prefer lighter skinned women" (p. 880). Hunter (1998) extended this research and determined that lighter skinned women were more likely to marry high status African American men than darker skinned African American women. These findings point to a relationship between African American self-esteem and skin tone.

\section{The Current Study}

Prior research on color consciousness (including that mentioned above) focused on the reanalysis of census-type data collected in the late 70s and early 80s (Hill, 2002; Hughes \& Hertel, 1990; Hunter, 2002; Keith \& Herring, 1991). This data was used to make inferences regarding differences in pre-existing life circumstances among African Americans. Other studies on color consciousness among African Americans addressed physiological and emotional issues such as body image, physical attributes and ethnic identity (Bond $\&$ Cash, 1992). The current study incorporates these research ideas while building on them in two unique ways. First, the data included in this study is current and was actively gathered from participants between 1999 and 2000. Second, the research format used in this study differed from previous studies in that the qualitative portion allowed participants to infuse a degree of personal experience into the discussion. The incorporation of qualitative methods for this type of research is supported by the work of other investigators who state that, "qualitative methodologies provide fuller, more detailed descriptions that are more exactly reflective of an individual's experience" (Schwitzer, Griffin, Ancis, \& Thomas, 1999). As such, the authors believe that the 
qualitative component of the current study facilitated the infusion of new perspectives to the powerful, yet limited empirical data available on the topic of color

consciousness.

Based on the previously mentioned ideas, the researchers wanted to extend the literature on the effects of skin tone on African American life circumstances via the following questions:

1. In what ways, if any, does skin tone influence an African American individual's annual income, level of educational attainment, racial identity and selfesteem?

2. Do African Americans prefer certain skin tones over others?

3. What characteristics do African Americans associate with their own and other African Americans' skin tones?

4. How does an African American's skin tone affect individual interpersonal and familial experiences?

\section{Method}

\section{Setting and Recruitment}

The settings for this study included 2 large cities, one in the Midwest and another in the Southeast. Participants in the Midwest were recruited from a large Midwestern university and the surrounding city via mailings, posted flyers and brief presentations by the principal investigator (PI) and three research assistants. Specifically, flyers were posted in all residence halls and many buildings throughout the campus and mailed to all African American undergraduate and graduate students on the campus. For the mailings, the PI enlisted the assistance of an administrative office on the campus that prepared mailing labels for all identified African American students on the campus. The PI then delivered sealed envelopes with the flyers enclosed to the administrative office. All letters were mailed in this manner to protect the students' confidentiality. In addition, the PI and 3 research assistants recruited participants via word of mouth requests. The PI recruited the participants from the southeast via word of mouth requests both via phone and in person. This recruitment method resulted in completed data for only two participants. Since the $\mathrm{n}$ was so small for the southeastern group, these participants were dropped from the analysis. All focus group participants were compensated with a catered dinner upon completion of the focus group.

\section{Participants}

Data was analyzed for 37 participants including 11 men (29.7\% of sample) and 26 women (70.2\% of sample). Age ranged from 19 to $78(\mathrm{M}=32.74, \mathrm{SD}=12.88)$ and household incomes ranged from 14,999 to 100,000 (Median $=47,042$ ). Participants were all high school graduates and over $90 \%(n=34)$ were college graduates. Regarding occupational status, $62 \%(\mathrm{n}=23)$ were graduate students, $5 \%(\mathrm{n}=2)$ were university faulty, $24 \%$ $(n=9)$ were employed in the public sector and $8 \%(n=3)$ were employed in the private sector.

\section{Measures}

The measures used in the study included, the Skin Color Questionnaire (SCQ) and the Skin Color Assessment Procedure (SCAP), both 3-item standardized measures of African American skin tone and associated attitudes toward skin tone designed by Bond and Cash (1992). The procedure for use of the SCAP was modified slightly for inclusion in this study. Specifically, rather than the original procedure in which participants selected a swatch of paint that most closely resembled their skin tone, participants selected the color that most closely matched their own from a color chart on a single sheet of paper. The color chart included a range of colors from dark (1) to light (9). The remaining portion of the SCAP remained as it was originally designed and included the following procedure. Individual participants were asked to select the color that most resembled their actual facial color; the color they would like to be; and finally, the color that the opposite sex finds most attractive. These ratings revealed participant perceptions of 1) actual skin tone 2) preferred skin tone and 3) opposite sex preferences for skin tone. In addition, the researchers assessed the participants' actual facial color using the color chart and these findings were then subjected to inter-rater reliability tests to determine the convergence between participant and researcher ratings of skin tone. Further, participant self-perceived skin color ratings were compared with the mean of the researcher ratings of participant skin color to determine the accuracy of participant self-ratings and participants' actual skin tone. Participants' SCAP ratings allowed for the calculation of discrepancy scores, which indicated the numerical difference between the selected ideal vs. the selfperceived skin color ratings. In addition, this final procedure was included to assess the accuracy of participants' ratings of personal skin color via a distortion rating.

For the Skin Color Questionnaire, the same color chart was used and participants were asked to answer the following three questions (response items are identified in parentheses): "How satisfied are you with the shade 
(lightness or darkness) of your own skin color?" ( 1 = extremely dissatisfied to $9=$ extremely satisfied); "Compared to most Black people, I believe my skin color is ..." $(1=$ extremely light to $9=$ extremely dark $)$ and "If I could change my skin color, I would make it ..." ( $1=$ much lighter, $5=$ exactly the same and $9=$ much darker). These items allowed the researchers to assess participant skin color satisfaction, self-perceived skin color (light-dark), and ideal skin color. In previous administrations of the SCQ and SCAP, the ranges used to assign respondents to skin tone categories respondents were 1-3 for light; 4-6 for medium and 7-9 for dark.

Two members of the research team developed a brief demographic questionnaire. This was an 8-item measure that identified basic socioeconomic and demographic information on participants and their families. The measure ascertained participant age, race, gender, occupational status, years of completed education, family income, parents' years of completed education and family of origin income during participant's childhood. No questions were included on this form to intentionally reveal participant identities.

The Rosenberg Self-Esteem Scale (Rosenberg, 1965) is a 10-item Likert scaled that was included to assess selfacceptance aspects of self-esteem. Responses were coded on a scale of 1 to 4 with 1 representing strong disagreement and 4 representing strong agreement. A total score was derived by summing all coded answers of the measure. Higher total scores indicated higher selfesteem. In previous studies employing this measure with African Americans, researchers reported Cronbach alpha reliability estimates ranging from .76 (Breland, Coleman, Coard, \& Steward, 2002) to .82 (Wade, 1996). Reliability analysis for this sample yielded a Cronbach's alpha of .78.

The Multigroup Ethnic Identity Measure (MEIM, (Phinney, 1992) is a 23-item scale that was included to examine three aspects of ethnic identity including attitudes towards one's own ethnic group, ethnic customs and behaviors, and commitment to one's own ethnic identity (Breland-Noble et al., 2003). In previous studies employing this measure with African American and other participants of color, researchers reported Cronbach alpha reliability estimates ranging from .64 (Longshore, 1999) to .82 (Branch, Tayal, \& Triplett, 2000). Reliability analysis for this sample yielded a Cronbach's alpha of 67 .

A qualitative research method was also used to collect data. Specifically, the researchers employed a focus group format. "Focus groups are in-depth discussions in which groups of between 8 and 12 people who share a particular set of characteristics or experiences are brought together, under the guidance of a facilitator, to discuss a topic of importance to a particular study "(Murray, 1998, p.313). Focus groups have been demonstrated to be especially useful in identifying problems that quantitative methods might miss such as personal accounts of experiences relevant to a topic or full explanations of ideas that cannot be garnered from closed-ended or multiple choice types of questions. In this study, African American participants were asked to describe their personal, familial, and community perceptions of and experiences in dealing with color consciousness. A semistructured interview guide format was used which allowed the investigator to gauge participant comfort levels and organize questions accordingly.

\section{Procedure}

Data collection occurred in single session 2-hour meetings. During the meetings, participants were briefed about informed consent via the dissemination of instructional letters further explicating the details of the research study. At that time only those participants who signed the consent form were included. The measures were administered during a single time period on one day, in the same sequential order. Upon completion of the measures, participants viewed a 22-minute video of the television program "Nightline" on the topic of color consciousness. Participants then engaged in an openended question format discussion that was facilitated by the PI and research assistants (note: the PI and research assistant who led the focus groups were both very dark skinned Black women). The questions included in the focus group discussion protocol are listed in Table 1.

The data obtained from these focus group sessions was audiotaped and transcribed (by an investigator not present for the focus groups). Data analysis was conducted by the PI using the constant comparative method coupled with thematic data analysis. The constant comparative method, "is an inductive process for forming a categorical model to describe the data collected in a study" (Schwitzer et al., 1999 p. 192). The constant comparative approach called for the PI to organize and reorganize the responses of the transcribed sessions until the data fit into the fewest, most meaningful categories possible.

Initially, one of the investigators produced handwritten copies of all audio-taped sessions and converted them into word-processed form. Next, a different investigator 


\section{Table 1: Questions for Semi-structured focus groups}

1) Tell us your first name and one thing you would like us to know about you.

2) What persuaded you to join the focus group?

3) When you hear the words "color consciousness", "colorism", and "colorstruck", what comes to mind?

4) In general do you agree or disagree with what was said in the show?

a) What one thing did you most agree with in the show?

b) What one thing did you most disagree with in the show? How has your experience differed from this one idea that you have identified as untrue for you?

5) Do you think the media (TV, Music Videos, and Film) affects the issue of color consciousness with African Americans? If so, in what way?

6) What is your earliest memory of discovering what skin tone/skin color you are?

7) Did your family discuss skin tone/skin color when you were a child? If so, give one example of things that were said.

8) What kinds of things do you remember hearing about your skin tone/skin color or that of your family as an adolescent/teenager? How did you feel when you heard these things?

9) What do you remember from your early adulthood experiences with peers about your skin tone/ skin color? How did you feel when these things happened?

10) In what ways do you think your own skin tone affects your life now (your career, professional/iob promotions, educational experiences/opportunities)?

11) Did the skin color of your significant other (husband/wife/partner) have any bearing on why you chose that person? If you do not yet have one, does the skin tone of a potential mate have any bearing on whether or not you choose that person?

created a master transcript of all focus group sessions in which responses were grouped according to their categorical "fit" with the questions asked. Any data that could not be categorized in this manner (i.e. that which did not directly address the stimulus questions) was organized in separate categories on the basis of categorical similarity. This system was used to code data into similar response categories. Then, the generally categorized data was reorganized into a smaller number of more specific categories. This process of creating smaller categories was replicated until the data could no longer be reorganized (i.e. no new themes emerged). The use of this method allowed the investigators to, "look for statements and signs of behavior that occur[ed] over time during the study," (Dye, Schatz, Rosenberg, \& Coleman, 2000 p. 2) with the goal of generating significant themes. This method generates themes that are, "generalized statements by respondents about beliefs, attitudes, values, and sentiments... [that] are culturally bound" (Murray, 1998, p.315). This model was especially useful given the multicultural focus of the study and the fact that the investigators were not interested in generating theory so much as in understanding an existing phenomenon (i.e. color consciousness) and providing a thematic model for illumination of the associated issues.

The investigators compared participants' self-ratings of skin tone and the mean ratings of the investigators' ratings of participant skin tone to assess the accuracy of the skin tone ratings. The acceptable inter-rater reliability of the researchers' 9-point ratings of participant skin color was indicated by a reliability coefficient of .81 .

Bivariate regression analyses were used to identify the effects of skin tone on self-esteem, ethnic identity, personal income and color consciousness. The researchers selected the bivariate regression analysis because the research in the area of skin tone as a power stimulus in African American life circumstances has not been comprehensively addressed in the literature. Therefore this type of basic statistical design was employed to assist in establishing a "baseline" of information on skin tone as a predictor/independent variable. Based on a power analysis, the researchers determined that a sample size of 30 was required to detect large effect sizes at the .05 significance level (Cohen, 1992). Note that a large effect size represents an effect that is noticeably larger than one visible to the naked eye of a careful observer, but not so large as to be trivialized (Cohen, 1992). In addition, paired sample t-tests were conducted to identify any differences among participants in their individually stated preferences for and individually stated perceptions of their own skin tones as measured by the SCAP and SCQ. T-tests were also computed to detect any gender differences in ethnic identity, self-esteem, and preferences for and perceptions of skin tone.

\section{Results}

\section{Overall perceptions of skin tone}

Table 2 presents the means and standard deviations for all studied variables.

Correlations were examined to answer the questions, "do African Americans prefer certain skin tones over others?" and "what characteristics do African Americans associate with their own and other African Americans' skin tones?" 
Table 2

Means and Standard Deviations for Variables Studied

\begin{tabular}{lcc} 
& MEAN & SD \\
\hline PHINNEY (Ethnic identity) & 3.64 & .29 \\
\hline MEANST (Raters' judgment) & 6.46 & 1.88 \\
\hline SCQ1 (Satisfaction) & 7.92 & 1.57 \\
\hline SCQ2 (perceived skin tone) & 5.31 & 1.67 \\
\hline SCQ3 (idealized skin tone) & 5.78 & 1.31 \\
\hline SCAPI (actual skin tone) & 6.41 & 1.87 \\
\hline SCAP2 (preferred skin tone) & 6.72 & 1.81 \\
\hline SCAP3 (opposite sex pref.) & 5.78 & 1.31 \\
\hline MOMEDUC (maternal education level) & 15.77 & 2.94 \\
\hline DADEDUC (paternal education level) & 14.61 & 3.66 \\
\hline EDUCLVL (participant education level) & 17.97 & 2.34 \\
\hline RSES (Self-esteem) & 37.70 & 3.37 \\
\hline INCOME & 44,135 & 28,600 \\
\hline AGE & 31.56 & 12.15 \\
\hline
\end{tabular}

* Note: The ranges for responses on the SCAP and SCQ were 1 - 9

Regarding skin tone accuracy ratings, the researchers ratings were identical for $41 \%(n=15)$ of the participants; within 1 point for $43 \%(n=16)$ of the participants and within 2 points for $11 \%(n=4)$ of the participants. Even though $5 \%(n=2)$ of the participants' ratings were greater than 3 points apart, this finding paralleled that of previous administrations of the SCAP (Bond and Cash, 1992) and suggested that participants viewed their own skin tones in a manner consistent with how others viewed their skin tones. Overall, the accuracy evaluations yielded a moderate positive correlation with participants' SCAP self-ratings $(\mathrm{r}=.50, \mathrm{p}=.002)$ and participants' SCQ lightness-darkness self-rating ratings $(\mathrm{r}=.43, \mathrm{p}=009)$. In addition, participants' self-ratings and lightness-darkness ratings of skin tone were highly positively correlated $(\mathrm{r}=$ $.73, \mathrm{p}=.000)$.
Regarding the participants' responses on the skin tone variables, results yielded a moderate positive correlation between participants' idealized skin tone and preferred skin tone $(\mathrm{r}=.50, \mathrm{p}=004)$ and between participants' selfperceived skin tone and idealized skin tone $(\mathrm{r}=.38, \mathrm{p}=$ .03). In addition, results revealed a moderate positive correlation between participants' preferred skin tone and self-perceived skin tone $(\mathrm{r}=.56, \mathrm{p}=.000)$.

Bivariate regression analysis indicated that as participant skin tones became darker, the stated wish to have darker skin also increased $(\mathrm{r}=.43) \mathrm{p}=.01$. This bivariate regression analysis indicated that $20 \%$ of the variance in personal skin tone preference was accounted for by the participants' skin tone.

The results of paired t-test analyses on the skin tone variables indicated that for all skin tone variables studied, statistically significant differences existed between the following pairs of variables: preferred skin tone and opposite sex preferences for skin tone $\mathrm{t}(36)=2.83, \mathrm{p}=$ .008; preferred skin tone and self-perceived (light/dark) skin tone $\mathrm{t}(36)=5.17, \mathrm{p}=.000$; and actual skin tone and self-perceived (light/ dark) skin tone $\mathrm{t}(36)=5.15, \mathrm{p}=.000$.

\section{Skin Tone, Ethnic Identity, and Socioeconomic Variables}

Regarding the question, "in what ways, if any, does skin tone influence an African American individual's annual income, level of educational attainment, racial identity and self-esteem?" results did not reveal any skin tone specific correlations, but did reveal a moderate positive correlation between participants' ethnic identity and years of completed education $(\mathrm{r}=.49, \mathrm{p}=.002)$ as well as ethnic identity and mother's completed years of education $(\mathrm{r}=.34, \mathrm{p}=.039)$. Participant incomes were moderately negatively correlated with their father's years of completed education $(\mathrm{r}=-.35, \mathrm{p}=.002)$ and their mother's level of completed education $(r=-.35, \mathrm{p}=.049)$. It is possible that this sample demonstrates a trend often found in African American families where successive generations strive to improve upon the socioeconomic circumstances of prior generations (McAdoo, 1997; Willie \& Reddick, 2003).

$\mathrm{T}$ tests were performed to examine any gender effects on self-satisfaction, preferred, idealized or opposite sex preferences for skin tone, ethnic identity and self-esteem. Table 3 presents the results for the significant findings.

The only significant difference found for the men and women was on the variable of self-esteem with women obtaining higher self-esteem scores than the men. 
Effects of Skin Tone on Life Circumstances and Experiences

The investigators discovered several emergent themes in examining the question, "how does an African

American's skin tone affect individual interpersonal and familial experiences?"

Overall, focus group members acknowledged that color consciousness exists and they provided data that contributed to generating the following emergent themes: 1) media reinforcement of negative aspects of color consciousness (via assigned /implied attributes) in portrayals of African Americans; 2) negative experiences in childhood and adolescence with color consciousness; 3 ) within family color consciousness; 4) mixed effects of skin tone on career/education; 5) negative effects of skin tone on romantic relationships 6) extensive nomenclature used in describing African American skin tones; 7) regional differences in color consciousness experiences; and 8) differential treatment by gender based on skin tone.

Media reinforcement of negative aspects of color consciousness. Respondents indicated that the media portrays African Americans in very stereotypical manners often showcasing light skinned women and dark skinned men as the aesthetic ideals. Many respondents shared the sentiments of a woman who said, "I I mean I know in terms of like music videos, um entertainment....and I always noticed how all the "pretty girls" were light skinned with long hair." And another who shared, " And I think that...when they show Tyson [Beckford, a model] when you look at the black man today, it's a chocolate hunk and so they're pushing it now."

Negative childhood and adolescent experiences with color consciousness. Many respondents reported being

\section{Table 3}

Means and $t$ Value from the Rosenberg Self-Esteem Scale

\begin{tabular}{lccc} 
Rosenberg & Men (11) & Women (26) & $\dagger$ \\
\hline Mean & 35.3636 & 38.6923 & 2.22 \\
\hline SD & 4.822 & 1.892 & \\
\hline Note: $p=.048$ & & & \\
\hline
\end{tabular}

espondents

indicated that the

media portrays

African Americans in

very stereotypical

manners often

showcasing light

skinned women and

dark skinned men as

the aesthetic ideals. ostracized by other African Americans because of the lightness or darkness of their skin. Female respondents in particular recalled being teased and not invited to play with other children because they were "too dark" or because they were light and perceived as haughty by their peers. Male respondents reported more teasing (e.g. being called negative names) than ostracism (i.e. having peers who refused to play or associate with them). Most respondents who recalled their first instances of discovering that others attended to their skin tone, reported that the awareness came during adolescence either in jr. high or high school.

Within family color consciousness. Two categories emerged in this area. Almost all respondents shared stories about personally hurtful experiences within their own families or recounted stories from friends' families. Others shared feelings of parental concern for children's present and future experiences. Many of the stories reflecting the former were focused on families enforcing the message that one should find a mate with the "right" (i.e. light) skin tone so that the offspring will be produced with the "right" color. In addition, respondents reported that at times their family members would actively make distinctions and associate attributes with family members of different skin tones. One respondent recounted how the light and dark skinned siblings within one family formed alliances based on skin tone (i.e. light vs. dark) and argued and fought regularly with no parental intervention. Two respondents reported hearing the name "Casper the Ghost" in reference to themselves or their infant children. The reference was used because in one respondent's words, "...[when] he (a very light skinned infant) put on the white things...he would fade away." Regarding parental concern, a mother reported that she knew her child might be both helped and harmed by her extremely light skin while other respondents reported that lighter skinned children within families were provided with preferential treatment and praised for being attractive while dark skinned children were routinely neglected.

Effects of skin tone on career/education. Respondents reported some advantages to having a lighter skin tone with regard to careers. A female respondent reported that she could not help but notice that of 5 women selected to earn a prestigious national academic fellowship, 4 of them (including herself) were very light skinned. Another woman echoed these sentiments when she stated, "...but every firm that I've been in and these are fairly large environments, most of the African Americans there have been fairly light complected [sic] and if they weren't light complected they had kind of like you know that 
questionable well maybe she could be [Black]".

Respondents also stated that they perceived light skinned men to reap some benefits from their light skin, yet the skin tone was bolstered by these individuals' abilities and hard work. Specifically, they spoke of the prevalence of color consciousness in the military, citing Colin Powell and other high ranking African American officials, as well as the negative perceptions directed toward dark skinned men in academic and private sector environments. Some of the darker skinned women reported that they believe themselves to be perceived as aloof and non-congenial because of their dark skin. One respondent captured the feelings of some of the participants when she stated, "I always found that as a darker skinned woman I was either expected, I felt like I was expected to be a little tougher." Others reported that they felt as if whites perceived them (dark skinned African American women) as too far outside the White standard to ever be able to assimilate to the norms of the workplace.

Negative affects of skin tone on romantic relationships. Two subcategories emerged within this larger area of color consciousness. Specifically, respondents reported actively selecting mates in an effort to reverse the deleterious effects of having their own skin tones and / or being hurt by others who engaged in this same process (of selecting mates by skin tone). Participants typically described how they had been hurt by a prospective mate's comments that he or she only dated people of a particular "hue" or how they were abandoned by a person of a particular skin tone and consequently chose to find partners at the opposite end of the skin tone spectrum. Overall, most female respondents reported assigning attributes to men based on skin tone with light skinned men being seen as significantly less virile than dark skinned men.

\section{Extensive nomenclature used in describing African American} skin tones. Not surprisingly, respondents indicated that an array of names exists for describing African Americans of differing skin tones. Most of the names that group members provided were familiar to all respondents, yet one ("Red-Bone") carried different connotations and was unfamiliar to some of the focus group participants.

Regional differences in color consciousness experiences. Group members strongly supported the idea that color consciousness exists in different forms depending on the region of the country in which one resides. Specifically, certain areas of the south were reported to have much more color consciousness than the north. In addition, respondents who had graduated from HBCUs (Historically Black Colleges and Universities) reported that they believed color consciousness to exist at greater levels than at PWIs (Predominantly White Institutions) where racism was more prevalent.

Differential treatment by gender based on skin tone. Almost all respondents agreed that the standards for men and women with regard to skin tone are very different. Specifically, many suggested that due to societal tendencies to judge women based on levels of attractiveness, there were fewer opportunities for women to view themselves through a cultural lens other than their attractiveness. Further, since media stereotypes reinforced a light-skinned ideal; lighter skinned women may reap the benefits of being light skinned. One respondent's words aptly capture the sentiments of the participants, "It seems as if... women and even little girls are sort of evaluated by their attractiveness... that you are more likely to run into...being evaluated by your hue more so than young boys [are]..." Other participants recalled that homecoming queens and the more popular girls in school were frequently very light skinned with European hair and facial features. Conversely, respondents reported that perceptions of African American boys were not as polarized and that often sports served as an equalizer such that extremely light or extremely dark skinned boys might be spared any teasing. Another interesting theme that emerged from the groups was associated with the "cycles" that characterize perceptions of African American men. Specifically, many of the men reported that at certain points throughout their development, a certain skin tone was considered "in." In other words, during certain periods in their development, light skinned men might be popular and positively perceived, while in other periods the opposite was true. Many of the male respondents and a few of the female respondents reported that depending on whom the popular media presented as prominent African American males at the time, skin tone preferences for men might move in the same direction. To paraphrase two respondents, "[the light skinned] brothers lost out in the mid 80's," and peers, "... would taunt him [a dark skinned sibling] and tease him and then... after Michael Jordan he was the best thing since cornbread."

\section{Discussion}

The findings appear to indicate that skin tone and color consciousness are very real phenomena that African Americans are aware of and can articulate the ways in which such issues affect their lives.

The researchers were interested in assessing African Americans' perceptions of their own and others' skin tones. The findings from this study strongly indicate that overall African Americans prefer their own skin tones to be in the middle range of colors $(M=6.72)$. Further, they believe that members of the opposite sex find mid-range, yet significantly lighter, skin tones to be most attractive $(M=5.78)$. Prior research supports the notion of preferences for mid-range skin tones, since many African Americans believe that those individuals in the middle of the skin tone spectrum do not experience as many 
traumas around skin tone as individuals at the polar ends might (Coard et al., 2001).

It appears that participants often stated a preference for moderately darker colors while harboring an ideal preference for a medium color. These findings reflect findings in other studies (Coard et al., 2001; Robinson \& Ward, 1995) in which participants indicated that those with medium skin fare better in life in general than African Americans at the poles of the skin tone spectrum. Therefore, it stands to reason that participants would idealize a skin tone that would afford them less opportunity to experience trauma.

As reported in previous work, African Americans do continue to harbor preferences for certain skin tones. Specifically, this sample indicated a selfpreference for almost dark skin, yet they idealized a significantly mid-range skin tone. A possible explanation for this finding might be associated with ethnic identity. Specifically, since the participants reported an overall high degree of ethnic identity $(M=3.64)$, it is possible that their stated preference for an almost dark color reflects their salient affiliations with being African American. Racial identity theory, as studied by authors like Helms, Carter and Cross, supports this idea of African Americans reporting an affinity for those things noticeably Black, like skin tone and facial features. Further, it is possible that given the generational status of the participants, with most being children of the 50s, 60's and 70s they were reared in the era of the Civil Rights and / or Black Power movements of the 60s and 70s. It was during these movements that African Americans espoused a strong ethnic identity and ties to their roots in Africa. As such, many African Americans witnessed the espousal of a Black beauty aesthetic that included traditionally "African" features such as dark skin and "full" facial features. In other words, it may be that the participants wished to demonstrate their commitment to the ethnic group by professing an almost dark preference while harboring an affinity for a medium skin tone. Leeds (1993) found similar results in her work with African American women, in that participants in her study reported a preference for darker skin and stated that they did not often make distinctions regarding color among their peers, yet upon further discussion revealed an idealization of lighter skin. As stated earlier, it is possible that within the African American community medium skin is preferred over dark skin due to the perceived and actual stresses often associated with very dark skin (Blair, Judd, Sadler, \& Jenkins, 2002; Breland, 1998; Hughes \& Hertel, 1990).

Regarding the effects of skin tone on annual income, acquired education, racial identity and self-esteem, the findings of this sample of African Americans indicated that skin tone alone did not explain current life circumstances. This finding is in stark contrast to other published research in which skin tone was demonstrated to be the power stimulus in predicting socioeconomic outcomes such as household income, job prestige, or marital desirability among African Americans (Hill, 2002; Hunter, 2002; Keith \& Herring, 1991). For this group, parental achievement and ethnic identity appeared to be power stimuli for their current socioeconomic status.

Regarding the effects that African Americans report skin tone to have on their life circumstances, Boyd-Franklin (1991) aptly described the pervasive nature of color consciousness within African American families and the current findings support her ideas. It appears that many participants learned about color consciousness via verbal and non-verbal family interactions. They also reported that the media reinforced most of the stereotypes that they learned from family and friends. Specifically, many participants recalled that light skinned African Americans (particularly women) were misperceived as haughty; dark skinned African American men misperceived as virile; light skinned African American men misperceived as impuissant; and dark skinned African American women misperceived as mean and/or aloof. It is possible that many African Americans in counseling may be aware of these stereotypes and may have or have been negatively impacted by them.

Culturally competent counselors and psychologists must be prepared to address color consciousness using a strong knowledge base and a delicate means of acknowledging the pain associated with this particular type of intraracial interaction.

With regard to dating and romantic relationships, an interesting trend emerged. Almost universally when discussing opposite sex preferences for skin tone, the African American male participants in the group stated that men they knew (not they themselves, but friends, family members, etc.) preferred very light skinned women with specific hair textures (i.e. long and Eurocentric). Interestingly though, 2 of the 12 men in the study openly professed an affinity for light skinned women at an earlier point in their development yet 
denounced it as a present-day occurrence. Conversely, the African American women in the groups reported self and other (friends, family members, etc.) preferences for very dark skinned men. Many of these women shared that they, "wanted someone who was darker because they associated darker with being stronger, a lot stronger and stuff". These findings are partially supported by the findings on the quantitative skin tone measures. Although the means for the men and women's perceptions of opposite sex preferences were in the medium range and not statistically different, female respondents reported that they perceive African American men to desire women with a lighter color $(\mathrm{M}=$ 5.22), while men stated that they perceive African American women to like men with a darker color $(\mathrm{M}=$ 6.12). This finding might speak to the possible correlations between media portrayals of African Americans and internalized preferences for opposite sex partners. Specifically, the participants in this study explained that the media portrayed African American women in such a way that light skinned women were viewed as objects of affection and that dark skinned men were viewed as strong and virile. Many participants shared that popular media representations of African American women include very light skinned women like actresses Halle Berry and Vanessa Williams, while popular media representations of African American men include very dark skinned males like actor Wesley Snipes and model Tyson Beckford. One focus group member's statement that regarding music videos, "...the only dark girls you ever saw were the ones dancing in the background [and] it was never the girl that the guys were chasing," expressed the sentiments of multiple participants. Further, many of the female African American participants shared that they were accustomed to seeing movies, advertisements and television programs that only showcased dark skinned African American men. As one participant put it, "that stereotype was broken in 1984 with Michael Jordan and with Nike and the Madison Avenue going ahead and exposing him to the rest of the world. That's how Tyson was able to get to where he is today." If dark skinned men and light skinned women appear to be sought after as objects of affection, what might this mean for light skinned African American men and dark skinned African American women? Findings from the focus groups revealed that, at least for African American women, dark skinned group members are left with emotional scars based on being overlooked for their lighter skinned counterparts (Hunter, 1998; Thompson \& Keith, 2001). Previous research has addressed this population briefly (BoydFranklin, 1991), but further research is certainly warranted to understand the effects of these occurrences on dark and light skinned African American women, and dark and light skinned African American men.

It is worth noting that most participants were initially reluctant to say that color consciousness exists within the African American community. It is possible that the presence of a white research assistant at some of the focus group meetings contributed to apprehension about sharing on this topic. It is also possible that the sample was reluctant to discuss intraracial discrimination when interracial discrimination occurs so frequently in their lives. Indeed, it was often necessary to redirect the participants in this sample to the topic of color consciousness among African Americans as it frequently drifted to racism and discrimination suffered at the hands of European Americans. The investigators hypothesize that some of this tangential discussion may be residue from the powerful and lasting effects of the black solidarity created by the Civil Rights Movement. Indeed, as one-fourth of the sample grew up in this period $(n=10)$ with the remaining participants being the children of people who grew up in this time period, it is possible that they learned the message of racism as a heinous evil and to not "air dirty laundry" by discussing the topic of Black-on-Black discrimination.

Some additional findings that warrant discussion are not necessarily focused on color consciousness, but are more associated with the sample's life experience of being African American. Recall that for all respondents there was a negative moderate correlation between their current incomes and their parents' completed levels of education. Since a majority of the participants were college graduates, and a significant number possessed graduate and professional degrees, it is possible that a message of subsequent generations doing better than those that came before them has been passed down. This notion is frequently discussed in the literature addressing African American families (Boyd-Franklin, 1991; Sue \& Sue, 1999). In addition, the moderate relationship that emerged between a participant's ethnic identity strength and a completed level of education speaks to the importance of being rooted in one's own culture as a buffer against racism and discrimination. Indeed, many participants stated during the focus groups that they used their strong sense of who they are as African Americans to help them make sense of and deal with the racism that they experienced regularly. This is an important finding for counselors who work with African Americans in that, given the current zeitgeist regarding the importance of assessing ethnic identity as an integral part of the counseling relationship (Helms \& Cook, 1999), it is essential that counselors who encounter African Americans with a strong ethnic identity incorporate this into any racism/discrimination interventions. Finally, it appears that African American mothers, at least for the participants in this sample, have done a good job of transmitting messages of ethnic pride and strength to their children. As noted, the participants in this sample demonstrated strong ethnic identities and these identities were moderately associated with their mothers' levels of 
education. Again these findings appear to reiterate previous findings in the literature that speak to the important of education and ethnic pride among African Americans (Sue \& Sue, 1999).

Overall, color consciousness appears to remain a prevalent and pervasive issue among African Americans as suggested by this small sample. The findings presented are extremely important and might be addressed by mental health professionals in counseling. It is possible that clinical prevention efforts with African American parents, where they are made aware of the messages about skin tone that can be transmitted to children, may assist in reversing the negative affects of color consciousness. It is also imperative for African Americans families to become aware of the ways in which the media assigns attributes to African Americans based on skin tone so that efforts can be developed to reverse the negative effects that are transmitted to group members. Counselors and psychologists can be helpful in these efforts by training African Americans to recognize, acknowledge and directly address these images both within and across racial groups. Counselors and psychologists who are not familiar with this idea should be aware of its potential to exist within African Americans families. Although it may be a sensitive subject to broach with clients, it can be very helpful for a culturally competent therapist to familiarize him or herself with the issue so that he or she may conduct an informed discussion on the topic with clients.

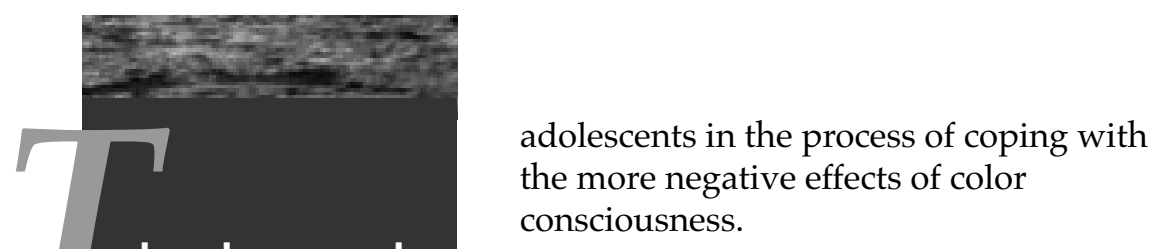

hey also reported

that the media

reinforced most of

the stereotypes that

they learned from

family and friends.

Specifically, many

participants recalled

that light skinned

African Americans

(particularly

women) were

misperceived as

haughty; dark

skinned African

American men

misperceived as

virile; light skinned

African American

men misperceived as

impuissant; and dark

skinned African

American women

misperceived as

mean and/or aloof.

\section{Limitations and Future Directions}

One of the most salient limitations to this study is the volunteer sampling procedure employed. It is possible that those

individuals for whom the issue of skin tone is salient were the ones most likely to agree to participate in the focus group. Therefore, this group may have devoted more thought to this topic than other African Americans. As such, their opinions may not be reflective of the general population, but more so of those African Americans who are well versed and educated in this topic in general. A second limitation to the study has to do with the skin tones of the African American investigators and assistants. As both African American data collectors were very dark skinned, it is possible that their presence was an impediment to the participants' ability to speak freely and to answer in an honest manner on the measures. Indeed, it is possible that the participants did not want to offend these researchers by being more frank in their discussions of darker skinned African Americans. Future research might include separating focus groups by gender and skin tone and matching these groups with moderators who reflect the compositions of the groups. Such methods might assist future investigators in eliciting more rich and multi-layered themes of color consciousness.

Regarding other future directions for research in this area, it is possible that given the African American women's

It also appears that counselors who work with African American adolescents should particularly be aware of the negative experiences that adolescents may have with this issue. Especially with regard to friendships and romantic relationships, it seems that African American children and adolescents may experience discrimination around being excluded as romantic partners or friends based solely on skin tone and conversely may be included as friends or romantic partners based solely on skin tone. Further, children and adolescents may be the objects of ridicule due to skin tone and, as described by multiple participants, such ridicule can be quite painful and have enduring effects. The findings indicate that group work with adolescents can be a very positive means of assisting African American reported attributions for African American men and African American men's reported overall lower levels of self-esteem, future research may explore the pervasiveness of these ideas separately and as they interact. For example, researchers might examine the nature and extent of African American women's stereotypes of African American men. In addition, future investigators might study the extent to which African American men are aware of the perceptions that African American women have of them and how these perceptions affect their (men's) psychological well being. In addition, it will be important to assess the extent to which skin tone is a factor in differential family treatment. Such research can greatly contribute to the 
literature on ameliorative interventions for use with African American families.

Finally the extent to which the media influences African Americans use of skin tone as a factor in selecting friends and romantic partners is an extremely worthwhile research area. This work can be especially useful for children and adolescents who are developing ideas regarding how they perceive themselves and others.

\section{References}

Altarriba, J., \& Bauer, L. M. (1998). Counseling the Hispanic client: Cuban Americans, Mexican Americans, and Puerto Ricans. Journal of Counseling \& Development, 76(4), 389-396.

Blair, I. V., Judd, C. M., Sadler, M. S., \& Jenkins, C. (2002). The Role of Afrocentric Features in Person Perception: Judging by Features and Categories. Journal of Personality \& Social Psychology July, 83(1), 5-25.

Bond, S., \& Cash, T. F. (1992). Black beauty: Skin color and body images among African-American college women. Journal of Applied Social Psychology, 22(11), 874-888.

Boyd-Franklin, N. (1991). Recurrent themes in the treatment of AfricanAmerican women in group psychotherapy. Women $\mathcal{E}$ Therapy, 11(2), 25-40.

Branch, C. W., Tayal, P., \& Triplett, C. (2000). The relationship of ethnic identity and ego identity status among adolescents and young adults. International Journal of Intercultural Relations, 24(6), 777-790.

Breland, A. M. (1998). A model for differential perceptions of competence based on skin tone among African Americans. Journal of Multicultural Counseling \& Development, 26(4), 294-311.

Breland, A. M., Coleman, H. L. K., Coard, S. I., \& Steward, R. J. (2002). Differences among African American Jr. High School Students: The Effects of Skin Tone on Ethnic Identity, Self-Esteem and CrossCultural Behavior. Dimensions of Counseling: Research, Theory and Practice, 30(1), 15-21.

Breland-Noble, A. M., Steward, R., Neil, D., Chan, C., Minami, T., Collins, W., et al. (2003). Biracial Individuals: Factors Affecting Ethnic Identification and Behaviors. Unpublished manuscript.

Coard, S. I., Breland, A. M., \& Raskin, P. (2001). Perceptions of and preferences for skin color, Black racial identity, and self-esteem among African Americans. Journal of Applied Social Psychology, 31(11), 2256-2274.

Codina, G., \& Montalvo, F. F. (1994). Chicano phenotype and depression. Hispanic Journal of Behavioral Sciences, 16(3), 296-306.

Cohen, J. (1992). A power primer. Psychological Bulletin, 112(1), 155-159.

Dye, J. F., Schatz, I. M., Rosenberg, B. A., \& Coleman, S. T. (2000). Constant Comparison Method: A Kaleidoscope of Data. Retrieved April 11, 2000, from http: / / www.nova.edu/ssss/QR/QR4-1/ dye.html

Edwards, O. L. (1972). Skin Color as a variable in racial attitudes of Black urbanites. Journal of Black Studies, 3(4), 473-483.

Graham, L. O. (1999). Our Kind of People. New York: HarperCollins.

Helms, J. E., \& Cook, D. A. (1999). Using race and culture in counseling and psychotherapy: Theory and process. Boston, MA: Allyn \& Bacon.-

Hill, M. E. (2002). Skin color and the perception of attractiveness among African Americans: Does gender make a difference? Social Psychology Quarterly, 65(1), 77-91.
Hughes, M., \& Hertel, B. R. (1990). The significance of color remains: A study of life chances, mate selection, and ethnic consciousness among Black Americans. Social Forces, 68(4), 1105-1120.

Hunter, M. L. (1998). Colorstruck: Skin Color stratification in the lives of African American women. Sociological Inquiry, 68(4), 517-535.

Hunter, M. L. (2002). "If you're light you're alright": Light skin color as social capital for women of color. Gender E Society, 16(2), 175-193.

Keith, V. M., \& Herring, C. (1991). Skin tone and stratification in the Black community. AJS - American Journal of Sociology, 97(3), 760-778.

Leeds, M. (1993). Young African American Women and the Language of Beauty. In K. A. Callaghan (Ed.), Ideals of Feminine Beauty (pp. 147159). CN: Greenwood Press.

Longshore, D. (1999). Help-Seeking by African American drug users: A prospective Analysis. Addictive Behaviors, 24(5), 683-686.

Maddox, K. B., \& Gray, S. A. (2002). Cognitive representations of Black Americans: Reexploring the role of skin tone. Personality \& Social Psychology Bulletin, 28(2), 250-259.

McAdoo, H. P. (Ed.). (1997). Black families. Thousand Oaks, CA: Sage Publications.

Murray, J. (1998). Qualitative Methods. International Review of Psychiatry, 10, 312-316.

Okazawa-Rey, M., Robinson, T., \& Ward, J. V. (1987). Black women and the politics of skin color and hair. Women \& Therapy, 6(1-2), 89-102.

Phinney, J. S. (1992). The multigroup ethnic identity measure: A new scale for use with diverse groups. Journal of Adolescent Research, 7(2), 156-176.

Robinson, T. L., \& Ward, J. V. (1995). African American adolescents and skin color. Journal of Black Psychology, 21(3), 256-274.

Rosenberg, M. (1965). Society and Adolescent Self-Image. NJ: Princeton University Press.

Sahay, S., \& Piran, N. (1997). Skin-color preferences and body satisfaction among South Asian-Canadian and European-Canadian female university students. Journal of Social Psychology, 137(2), 161-172.

Sandler, K. (1994). Finding a space for myself in my film about color consciousness. In D. Willis (Ed.), Picturing Us: African American identity in photography. New York: W.W. Norton and Company.

Scales-Trent, J. (1995). Notes of a White Black woman: Race, color and community. University Park, PA: Pennsylvania State University Press.

Schwitzer, A. M., Griffin, O. T., Ancis, J. R., \& Thomas, C. R. (1999). Social adjustment experiences of African American college students. Journal of Counseling $\mathcal{E}$ Development, 77(2), 189-197.

Seltzer, R., \& Smith, R. C. (1991). Color Differences in the Afro-American community and the differences they make. Journal of Black Studies, 21(3), 279-286.

Sue, D. W., \& Sue, D. (1999). Counseling the culturally different: Theory and practice (3rd ed.). New York: Wiley.

Telles, E. E., \& Murguia, E. (1990). Phenotypic discrimination and income difference among Mexican Americans. Social Science Quarterly, 71(4), 682-696.

Thompson, M. S., \& Keith, V. M. (2001). The blacker the berry: Gender, skin tone, self-esteem and self-efficacy. Gender \& Society, 15(3), 336357.

Wade, T. (1996). The relationships between skin color and self-perceived global, physical, and sexual attractiveness, and self-esteem for African Americans. Journal of Black Psychology, 22(3), 358-373.

Willie, C. V., \& Reddick, R. J. (2003). A new look at Black families (5th ed.).Walnut Creek, CA: Altamira. 